\title{
Sistema de mitigación de polvo de mineral de hierro en el área de chancado del sector de San Nicolás-Marcona
}

CARlos YABAR Baños ${ }^{1}$ Vidal Aramburú Rojas ${ }^{2}$ FÉlix TiNOCO ÁNGELES ${ }^{3}$

Recibido: 21/08/2019 Aceptado: 06/11/2019 Publicado: 16/10/2020

\begin{abstract}
RESUMEN
El objetivo de esta investigación es demostrar la disminución en la polución ambiental y exposición ocupacional mediante la sistematización del proceso de mitigación de polvo de hierro en el área de chancado del sector de beneficio San Nicolás de la empresa minera Shougang Hierro Perú S. A. A. El estudio se desarrolló bajo la metodología de tipo aplicativo, llegándose a demostrar, mediante monitoreo ambiental de partículas suspendidas, la relación causal entre polución ambiental y exposición ocupacional. Con una muestra de 15 trabajadores, se logró medir la percepción de disminución de la polución ambiental con un enfoque cuantitativo y un procesamiento de datos con el método de estadística descriptiva e inferencial. Los resultados reflejan que el promedio del nivel de influencia del esquema de mitigación de polvo de mineral de hierro es significativo, a un nivel de confianza del $95 \%$ y significancia del $5 \%$; en tal sentido, el sistema es eficiente para la reducción hasta el límite máximo permisible de calidad ambiental actual $\left(500 \mu \mathrm{g} / \mathrm{m}^{3}\right)$, el límite de la exposición ocupacional a partículas de polvo respirable $\left(3.2 \mathrm{mg} / \mathrm{m}^{3}\right)$ y de polvo inhalable $\left(13 \mathrm{mg} / \mathrm{m}^{3}\right)$. Luego de los monitoreos ambientales y ocupacionales respectivos, se pudo lograr un límite máximo permisible de calidad ambiental menor a $220 \mu \mathrm{g} / \mathrm{m}^{3}$, un límite ocupacional de partículas de polvo respirable de $2.2 \mathrm{mg} / \mathrm{m}^{3}$ y de polvo inhalable de $9.2 \mathrm{mg} /$ $\mathrm{m}^{3}$ en el área de chancado del sector de beneficio San Nicolás. Así, se concluye que el sistema de mitigación de polvo de hierro es eficiente para minimizarlo en la planta indicada.
\end{abstract}

Palabras clave: mitigación; polvo; planta chancadora; salud ocupacional; seguridad ocupacional.

\section{INTRODUCCIÓN}

Lo minero, a nivel general, es visto como algo dañino y atrofiante hacia el medio ambiente, pues produce perturbaciones de salud en las comunidades. Actualmente, el desarrollo tecnológico permite el uso de elementos para minimizar esta situación. Esto viene como consecuencia de las exigencias de la población y de un mundo globalizado, en el que se busca un progreso menos contaminante.

Este estudio se ha localizado en una planta chancadora del sector de beneficio San Nicolás de Shougang Hierro Perú S. A. A., donde se observó la generación de material particulado. Ante esta situación, se hacía necesaria la adaptación de un medio para disminuir la cantidad de moléculas presentes en el ambiente (especialmente, en las cercanías de la planta) mediante la colocación de un método que absorba esta nube de partículas.

Las referencias para realizar dicha tarea han sido el Decreto Supremo n. ${ }^{\circ}$ 007-2008-MINAM (García y Fernández, 6 de diciembre de 2008), las normativas para la realización de monitoreos ambientales detalladas en el Decreto Supremo n. ${ }^{\circ}$ 059-93-EM (Ministerio de Energía y Minas, 10 de diciembre de 1993) y la nueva Ley de Seguridad y Salud en el Trabajo n. ${ }^{\circ} 29783$ en 2011 (Humala y Villena, 20 de agosto de 2011; Humala y Villena, 25 de abril de 2012).

Las partículas sólidas de polvos son generadas en las diferentes operaciones de la minería, en las que se produce una serie de emisiones de polvo en la atmósfera; fundamentalmente, esto sucede durante las actividades extractivas, como la voladura, el

1 Ingeniero de materiales por la Universidad Nacional San Agustín de Arequipa (Arequipa, Perú) Actualmente, labora como prevencionista de control de pérdidas en la empresa Shougang Hierro Perú S. A. A. (Ica, Perú).

ORCID: https://orcid.org/0000-0003-0854-6852

E-mail: cyabar@shp.pe

2 Doctor en Gestión de Empresas y magíster en Ingeniería Metalúrgica por la Universidad Nacional Mayor de San Marcos (Lima, Perú). Actualmente, es docente de la Facultad de Ingeniería Geológica, Minera, Metalúrgica y Geográfica de la misma universidad. (Lima, Perú). ORCID: https://orcid.org/0000-0001-7411-3866 E-mail: varamburu@unmsm.edu.pe

3 Magíster en Gestión de Operaciones y Servicios Logísticos por la Universidad Nacional Mayor de San Marcos (Lima, Perú). Consultor independiente. ORCID: https://orcid.org/0000-0002-2630-4657 E-mail: tinocofelix@gmail.com 
carguío, el acarreo, el chancado primario o secundario de mineral y el transporte por fajas. Mediante las supervisiones ambientales PM-10, se registran concentraciones medias aritméticas diarias superiores a $350 \mu \mathrm{g} / \mathrm{m}^{3}$, las cuales representan el límite máximo de calidad del aire aprobada por la Resolución Ministerial n. ${ }^{\circ}$ 315-96 EM/VMM (Hokama, 16 de julio de 1996) (ver Tabla 1).

Un aspecto ambiental de especial consideración es la velocidad del viento que se registra en el distrito de San Juan de Marcona, provincia de Nazca, departamento de Ica, considerado como uno de los lugares con mayor incidencia de vientos a nivel nacional, con un promedio de $9 \mathrm{~m} / \mathrm{s}$ a un nivel de $40 \mathrm{~m}$ s. n. m. (Glave y Kuramoto, 2002). Este es un factor influyente a considerar en el proceso de mitigación de polvo en la planta de chancado secundario, ubicada a 3200 m s. n. m.

\section{Efectos patológicos del polvo en la minería}

Para Martínez, Quero, Isidro y Rego (2001), estas nubes de partículas, en principio, debido a su composición, disminuyen la iluminación ambiental, ocasionando daños en la salud. Este efecto ocurre por el tamaño de las partículas y por la combinación de distintos elementos. Las partículas tienen diferentes tamaños, lo que les permite mantenerse en el aire, por efecto de la fuerza de sustentación, donde las de menor dimensión y densidad son arrastradas por mayores recorridos y tiempo. Una vez que el viento baja su velocidad, las partículas tienden a caer al suelo.

Para constituyentes de poca densidad y dimensiones inferiores a $2.3 \mathrm{mg} / \mathrm{m}$, las partículas se mantienen por un tiempo relativamente mayor en el aire, lo que permite que sean inhaladas por los seres vivos, produciendo efectos sobre su salud. Cuando las partículas están por debajo de un valor de 10 $\mathrm{mg} / \mathrm{m}$, son absorbidas de manera más fácil y continua, con la posibilidad de producirse perturbaciones de alcance significativo.
El valor del límite máximo permisible para conservar la calidad del aire, dispuesto por la normativa nacional, es de $350 \mu \mathrm{g} / \mathrm{m}^{3}$ para las partículas en suspensión. Para una concentración media aritmética diaria, mediante los monitoreos PM-10 realizados en nuestro caso, se determinó que la concentración supera ese límite, lo que evidencia la necesidad de medidas de control para la optimización del aire.

\section{Escogimiento y dotación de equipos para la disminución de elementos de polución}

Según los estudios realizados por Warner (1981) y Anguita y Moreno (1993), las cribas están a un espaciamiento menor para poder filtrar partículas muy pequeñas contenidas en el aire. La captación de partículas suspendidas de polvo dependerá directamente del tamaño de las mismas y la aplicación posterior de un equipo que integre el sistema de mitigación de polvo (ver Tabla 2).

Además de lo anterior, Vega (2007) refiere a qué equipos usar en función de la retención de las partículas contaminantes. Para dicha elección, no es suficiente lo físico en cuanto a tamaño, forma o cualquier otra especificidad de las partículas, lo importante es su composición química, determinante en lo dañino y perturbador para la salud.

\section{Sistema de colectores de polvo}

El trabajo de Moraga, Rivera y Soto (2013), en Codelco, Chile, aborda el caso de una planta de chancado secundario-terciario; además, hace la descripción de 19 colectores de polvo operativos, de los cuales, 13 son del tipo Aeromix Wet Scrubbers y 6 , del tipo Rotoclone. Estos equipos para sacar el polvo se encuentran en lugares donde pueden absorber las partículas de manera continua y permanente de los medios que transportan el material triturado. Este polvo atrapado es mezclado con agua para que se aglomere y pueda ser vertido en los canales de desagüe, de donde, luego, separado del agua,

Tabla 1. Niveles máximos permisibles de calidad de aire.

\begin{tabular}{|l|c|c|c|}
\hline \multicolumn{1}{|c|}{ Parámetro } & $\begin{array}{c}\text { Concentración media } \\
\text { aritmética diaria } \\
\mu \mathrm{g} / \mathrm{m}^{3}(\mathrm{ppm})\end{array}$ & $\begin{array}{c}\text { Concentración media } \\
\text { aritmética anual } \\
\mu \mathrm{g} / \mathrm{m}^{3}\end{array}$ & $\begin{array}{c}\text { Concentración media } \\
\text { geométrica anual } \\
\boldsymbol{\mu g} / \mathrm{m}^{3}\end{array}$ \\
\hline Anhídrido sulfuroso & $572^{*}$ & 172 & - \\
\hline Partículas en suspensión & $350^{*}$ & - & 150 \\
\hline Plomo & - & 0.5 & - \\
\hline Arsénico & 6 & - & - \\
\hline
\end{tabular}

* No debe ser excedido más de una vez al año.

Fuente: Hokama (16 de julio de 1996). 
Tabla 2. Tamaños de partículas a ser atrapadas.

\begin{tabular}{|l|c|}
\hline \multicolumn{1}{|c|}{ Equipo } & Rango de partículas que atrapa en micras \\
\hline $\begin{array}{l}\text { Precipitadores electrostáticos } \\
\text { Torres empacadas }\end{array}$ & 0.01 a 90 \\
\hline Filtros de papel & 0.01 a 100 \\
\hline Filtros de tela & 0.005 a 8 \\
\hline Lavadores de gases & 0.05 a 90 \\
\hline Separadores centrífugos & 0.05 a 100 \\
\hline Cámaras de sedimentación & 5 a 100 \\
\hline
\end{tabular}

Fuente: Vega (2007)

se recoge el material depositado. Este cúmulo de minerales es recuperado para su procesamiento.

Esta agrupación de extractores, en general, está en continuo funcionamiento, es por ello que debe tener un plan de mantenimiento programado para evitar paradas no deseadas que puedan afectar la succión de las moléculas de polvo y generar una polución de gran alcance. A su vez, es necesario llevar un registro del uso de estos equipos a través de un personal calificado (Moraga et al., 2013).

Las propiedades físicas y químicas de las partículas son invariables. El tamaño, forma y particularidades de las mismas están sujetas a la cantidad de humedad presente en el material, a los equipos usados y su mantenimiento. Es importante considerar la cota de las acumulaciones de material y, de manera expresa, de las condiciones climatológicas entre ellas: cantidad de agua caída por precipitaciones, variabilidad de la dirección y sentido de la celeridad del viento.

Es importante destacar a Martínez et al. (2001), quienes hacen la diferenciación relativa desde el tamaño, dado que, independientemente de este, la salud de los trabajadores se deteriora, pues las partículas se alojan en el sistema pulmonar: las de mayor tamaño van a la tráquea, ductos nasales y bronquios; las intermedias, a los sectores pulmonares y las extrafinas, a la intimidad alveolar.

\section{Valores admisibles de polvos que contami- nan el aire}

Los patrones actuales de aceptación de niveles de contaminación de aire son usados referencialmente como aquellos que tienen poca capacidad de generar problemas de salud. Se encuentran señalados en los valores de los Estándares de Calidad Ambiental (ECA) para el aire, establecidos por el Ministerio de Ambiente. Estos valores deben ser considerados como primarios para desarrollar políticas ambientales y proyectos de tipo minero o de otra clase.
Por su lado, la EPA (Environmental Protection Agency) de EE. UU. estima datos de polución del aire, y refiere a límites de la misma para niños, ancianos y asmáticos, en primera instancia; para el descenso de la visión; así como para evitar perturbaciones de la flora y fauna (Bartual y Berenguer, 2001).

\section{Contaminación ambiental}

Según Aguilar (2006), la propagación del daño del medio ambiente es producto de diversos agentes presentes en el aire, agua y en partes donde las condiciones de vida están amenazadas por elementos tóxicos, sean estos de tipo bioquímico, biofísico o simplemente físico o químico; además, es posible que se presenten uno de ellos o todos en conjunto. De cualquier manera, todos estos elementos afectan el desenvolvimiento humano e incrementan el desmejoramiento de la flora y la fauna. Campos (2000) complementa la definición anterior manifestando la existencia de los factores bióticos, aquellos de los seres vivos y sustancias orgánicas, y los no bióticos, mencionados inicialmente (agua, aire y minerales), que afectan de algún modo el medio ambiente.

\section{Pasivos ambientales de la minería}

Según Herrera y Millones (2011), el consumo de agua es un componente que perturba de forma constante el desarrollo agrícola y pecuario de las zonas cercanas a los centros trituradores de material. Este agotamiento está ligado a lavar las cantidades de movido y removido, ya que dicha agua relativamente contaminada afecta las cuencas hidrográficas del Locumba, Cañete, Mantaro, Acarí y Moche, además, una parte de estas llega a la vertiente del Pacifico. Medina (2008) acota que en el río Mantaro el efecto de las aguas usadas en el lavado de material ha ocasionado pérdidas de animales y tierras cultivables, y daños significativos a la economía de la zona. La empresa involucrada en dichos detrimentos señala que, en estos momentos, el caudal de este río solo sirve para desagüe 
de las plantas cercanas a este afluente. En esta misma línea, se debe mencionar el daño ecológico a la Reserva Nacional de Junín, en el lago Chinchaycocha, situación que ha contribuido a la eliminación de animales, ríos y plantas, y al desmejoramiento de la calidad del aire; asimismo, ha promovido la desaparición de especies como el zambullidor de Junín y otras aves presentes en el lago (Castillo, 2008).

\section{METODOLOGÍA}

\section{Implementación del sistema de atenuación de} polvos en el área de chancado $n .^{\circ} 1$-San Nicolás

Las tareas de supervisión y monitoreo de los ecosistemas se llevan a cabo con el objetivo de cuantificar la existencia y concentración de contaminantes en el ambiente, además, permiten examinar la situación en que se conservan los recursos naturales (Organismo de Evaluación y Fiscalización Ambiental, 2014).

Las condiciones generales fueron: $83.5 \mathrm{~m} \mathrm{~s}$. n. m., temperatura máxima del aire: $32{ }^{\circ} \mathrm{C}$, temperatura mínima del aire: $18{ }^{\circ} \mathrm{C}$, humedad relativa: $95 \%$ y atmósfera salina (ver Figura 1).

\section{Descripción del proyecto}

La ejecución consistió en lo siguiente:

- Instalación de ductos colectores, campanas de extracción y soportes metálicos desde la línea 1 y la torre de zarandas.
- Montaje de precipitador.

- Implementación de transportadores que recolectan partículas finas.

- Montaje de ventilador.

- Montaje de chimenea.

- Construcción de pozas de humidificación.

- Montaje e instalación de compresora

- Montaje e instalación de "tanque pulmón" para almacenar aire de $3 \mathrm{~m}^{3}$.

- Montaje de línea de inyección de aire para limpiar filtros.

- Instalación de sistemas de inicio, control y equipamiento auxiliar.

- Montaje e instalación del equipamiento eléctrico en media tensión (celdas y/o arrancadores en $4.16 \mathrm{kV})$ y en baja tensión (440 V, $220 \mathrm{~V}, 120 \mathrm{~V})$.

- Montaje e instalación de infraestructura eléctrica y sistemas de canalización para el funcionamiento y supervisión de diferentes equipos electromecánicos.

- Instalación de infraestructura eléctrica de fuerza, instrumentación, automatización y de monitoreo en general.

- Construcción de subestación eléctrica completamente equipada.

En la Tabla 3, se muestran los parámetros técnicos considerados en la empresa para la implementación del sistema.

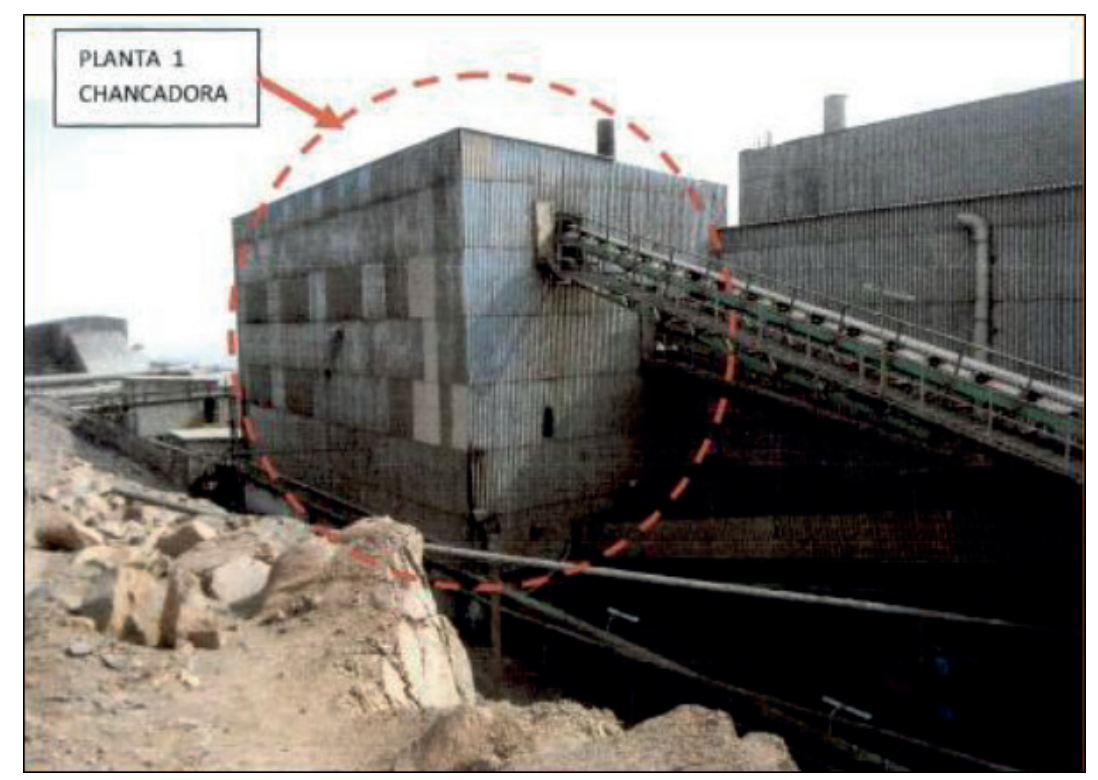

Figura 1. Área de chancado de Shougang Hierro Perú S. A. A. Fuente: Elaboración propia. Fotografía de Carlos Yabar Baños. 
Tabla 3. Parámetros técnicos. Shougang Hierro Perú S. A. A.

\begin{tabular}{|l|c|}
\hline \multicolumn{1}{|c|}{ Parámetro } & Unidad \\
\hline Volumen de aire procesado & $450000 \mathrm{~m}^{3} / \mathrm{h}$ \\
\hline Área de filtrado & $7200 \mathrm{~m}^{2}$ \\
\hline Velocidad del viento en filtro & $1.04 \mathrm{~m} / \mathrm{min}$ \\
\hline Resistencia de equipo & $1800 \mathrm{~Pa}$ \\
\hline Número de cámara de filtros & 16 \\
\hline Cantidad de mangas de filtros & 2400 \\
\hline Dimensiones de mangas de filtros & $0160 \times 6000 \mathrm{~mm}$ \\
\hline Dimensiones de soporte de mangas de filtros & $0155 \times 5980 \mathrm{~mm}$ \\
\hline Cantidad de soportes para filtros de manga & 2400 \\
\hline Material de los filtros & Waterproof Polyester Needle Felt \\
\hline Cantidad de válvulas de pulso & 160 \\
\hline Dimensiones de válvulas de pulso & $3 "$ \\
\hline Dimensiones de válvulas Flashboard & $300 \times 300$ \\
\hline Cantidad de válvulas de descarga & 8 \\
\hline Tipo de válvula de descarga & YJD-16A \\
\hline Cantidad de válvulas Off-line & 16 \\
\hline
\end{tabular}

Fuente: Elaboración propia.

\section{RESULTADOS Y DISCUSIÓN}

Se ha encontrado personal expuesto a los polvos o materiales particulados generados en las diversas plantas de chancado secundario, equivalente a 15 trabajadores. Asimismo, se ha monitoreado al personal que labora en el interior de la planta, esto incluye al personal de mantenimiento mecánico y eléctrico, y los operadores de equipos de planta. El punto de monitoreo mensual con los equipos instalados PM10 fue en lugares estratégicos aprobados por el Programa de Adecuación y Manejo Ambiental (PAMA).

La selección de la muestra se ha realizado de forma paulatina: se inició con las personas con mayor exposición hasta llegar a aquellas con menor exposición. Se registró cada monitoreo y se ha determinado la comparación con registros anteriores.

Supervisión ambiental PM10. Su finalidad es la evaluación de las minúsculas partículas de polvo, en sólido o líquido, las cenizas, el hollín, las partículas metálicas y el cemento, los cuales se hallan en estado de dispersión en el espacio atmosférico y cuyo radio es menor a $5 \mu \mathrm{m}$. En su composición se presentan sustancias inorgánicas, como sales de aluminio y sílice, metales pesados, entre otros. El particulado orgánico se asocia a polvo de carbono.

Monitoreos personales de polvo respirable e inhalables con bombas SCORT MSA. Es el estudio que se lleva a cabo para establecer una comparación con la metodología de muestreo $\mathrm{NIOSH}$, e intenta encontrar la magnitud de concentración de polvo que puede ser respirado en el lugar de trabajo (vía el método 0600, que denota las partículas no regulables respirables).

Estación meteorológica. Es un dispositivo cuya finalidad es medir y detectar regularmente diferentes variables meteorológicas. Los datos que se obtienen se emplean tanto para la predicción, a partir de modelos numéricos, como para investigaciones climatológicas.

Mapas de riesgo. Es el mapa de la planta chancadora con la identificación de señales de advertencia, informativas, de obligatoriedad y contra incendios.

Matrices IPERC de línea base. Es la tabla para identificar peligros, evaluación de riesgos y controles.

Las Tablas 4 y 5 reportan los resultados del monitoreo de polvos suspendidos entre junio y octubre de 2016 , con equipo de alto volumen.

A continuación, se ilustran los resultados obtenidos en la evaluación postest al medir las cantidades registradas de la polución ambiental. Esto se realizó mediante un cuestionario aplicado a los 15 trabajadores que desarrollaban sus labores en la planta chancadora de San Nicolás de la empresa Shougang Hierro Perú S. A. A. (ver Tabla 6), con 


\begin{tabular}{|c|c|c|c|c|c|c|}
\hline बఫ़" & ల్ల్ & $\stackrel{\stackrel{R}{\sim}}{\leftarrow}$ & I & $\stackrel{\infty}{\varpi}$ & 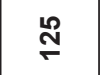 & ๑ \\
\hline 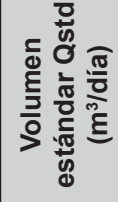 & $\begin{array}{l}\hat{0} \\
\stackrel{0}{0} \\
\stackrel{2}{\leftarrow}\end{array}$ & 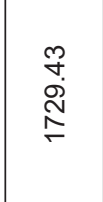 & 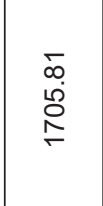 & $\begin{array}{l}\stackrel{\infty}{m} \\
\stackrel{N}{N} \\
\stackrel{N}{N}\end{array}$ & 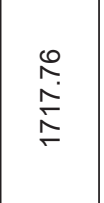 & $\begin{array}{l}\stackrel{\llcorner}{\circ} \\
\stackrel{N}{N} \\
\stackrel{5}{L}\end{array}$ \\
\hline 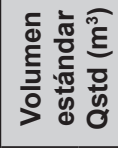 & 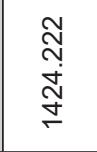 & 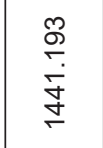 & 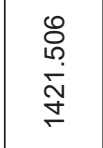 & 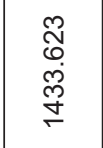 & 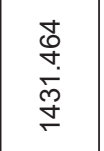 & 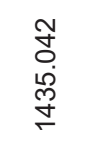 \\
\hline 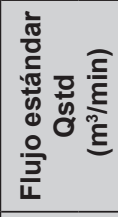 & 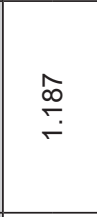 & 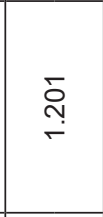 & $\stackrel{\stackrel{\infty}{\infty}}{\stackrel{\infty}{\leftarrow}}$ & $\stackrel{\stackrel{\Omega}{\circ}}{\check{\leftarrow}}$ & $\stackrel{\mathscr{\Omega}}{\leftarrow}$ & $\stackrel{\mathscr{Q}}{\stackrel{9}{\leftarrow}}$ \\
\hline 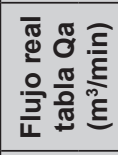 & $\stackrel{\infty}{\stackrel{\infty}{\rightleftarrows}}$ & $\stackrel{\infty}{\stackrel{\infty}{\leftarrow}}$ & 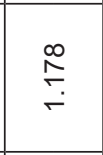 & $\stackrel{\infty}{\stackrel{\infty}{\leftarrow}}$ & $\stackrel{\infty}{\stackrel{\infty}{\leftarrow}}$ & $\stackrel{\stackrel{\varrho}{\circ}}{\leftarrow}$ \\
\hline 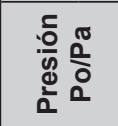 & $\begin{array}{l}\stackrel{+}{0} \\
\stackrel{\circ}{0} \\
0\end{array}$ & $\begin{array}{l}N \\
\hat{a} \\
0\end{array}$ & 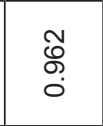 & $\begin{array}{l}\widetilde{N} \\
\stackrel{5}{\circ} \\
0\end{array}$ & 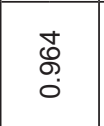 & $\begin{array}{l}\stackrel{8}{\circ} \\
\stackrel{0}{\circ}\end{array}$ \\
\hline 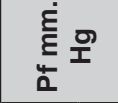 & 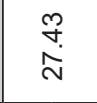 & $\stackrel{\stackrel{8}{+}}{\dot{N}}$ & $\begin{array}{l}\stackrel{0}{\llcorner} \\
\stackrel{\infty}{\infty} \\
\stackrel{\infty}{N}\end{array}$ & $\begin{array}{l}\infty \\
\stackrel{\infty}{ } \\
\stackrel{\infty}{\infty}\end{array}$ & $\stackrel{\stackrel{n}{N}}{\stackrel{N}{N}}$ & $\begin{array}{l}\overline{0} \\
\stackrel{\tilde{j}}{ }\end{array}$ \\
\hline 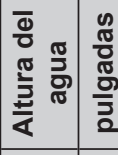 & 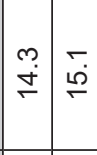 & $\stackrel{\circ}{\stackrel{\circ}{F}} \stackrel{\stackrel{0}{F}}{F}$ & \begin{tabular}{l|l}
0 & 0 \\
$\stackrel{\rho}{\circ}$ & $\stackrel{0}{\leftarrow}$ \\
$\stackrel{\rho}{\circ}$
\end{tabular} & 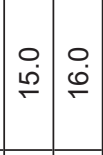 & 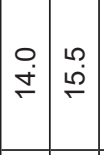 & 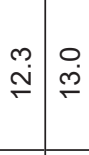 \\
\hline 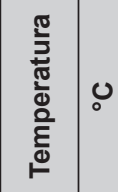 & 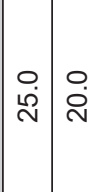 & \begin{tabular}{l|l} 
& $\circ$ \\
$\stackrel{i}{N}$
\end{tabular} & \begin{tabular}{l|l}
$\stackrel{0}{\sim}$ & $\stackrel{\sim}{\sim}$ \\
$\stackrel{\sim}{\sim}$
\end{tabular} & 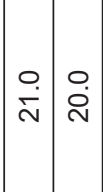 & \begin{tabular}{l|l}
0 & 0 \\
$\dot{\sim}$ & $\stackrel{\dot{d}}{\sim}$
\end{tabular} & $\stackrel{\circ}{\stackrel{\leftrightarrow}{\sim}} \stackrel{\circ}{\stackrel{\dot{m}}{N}}$ \\
\hline 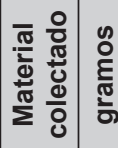 & $\begin{array}{l}\infty \\
\stackrel{N}{0} \\
\stackrel{0}{0}\end{array}$ & 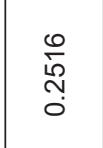 & $\begin{array}{l}\text { o } \\
\text { f } \\
0 \\
0\end{array}$ & $\begin{array}{l}\stackrel{0}{0} \\
\stackrel{0}{0} \\
0\end{array}$ & $\begin{array}{l}\frac{\mathscr{R}}{2} \\
\stackrel{0}{0}\end{array}$ & 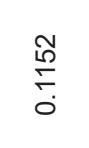 \\
\hline 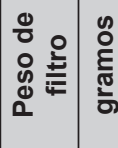 & 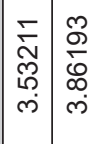 & 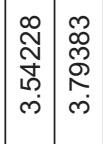 & 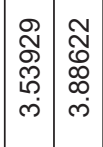 & 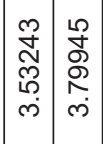 & 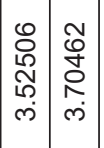 & 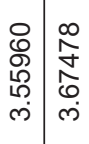 \\
\hline 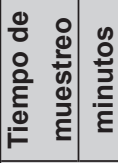 & ్ㅗ & 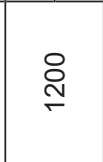 & 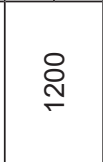 & 옴 & $\stackrel{\stackrel{ }{ }}{\sim}$ & 오 \\
\hline$\stackrel{\mathscr{0}}{\Sigma}$ & & 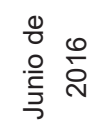 & & & 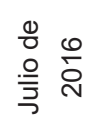 & \\
\hline$\stackrel{\circ}{\stackrel{ }{\underline{L}}} \stackrel{\circ}{\mathrm{L}}$ & $\stackrel{\leftrightarrow}{\sim}$ & $\underset{N}{N}$ & $\stackrel{\mathbb{N}}{\stackrel{1}{*}}$ & $\stackrel{\infty}{N}$ & ৪্ল & ర్లి \\
\hline $\begin{array}{l}z \\
\frac{0}{0} \\
\mathbb{5} \\
\mathbb{4}\end{array}$ & \multicolumn{6}{|c|}{ ஸ் } \\
\hline
\end{tabular}

\begin{tabular}{|c|c|c|c|c|c|c|}
\hline हू & 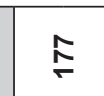 & $\stackrel{\mathscr{m}}{\sim}$ & 오 & J & $\stackrel{\varrho}{\stackrel{N}{N}}$ & న̊ \\
\hline 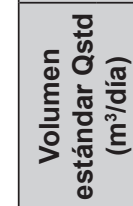 & $\begin{array}{l}\tilde{O} \\
\stackrel{\infty}{0} \\
0 \\
0\end{array}$ & 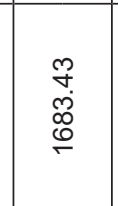 & 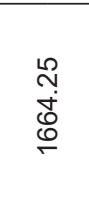 & $\begin{array}{l}\hat{0} \\
\stackrel{0}{0} \\
\stackrel{0}{0}\end{array}$ & $\begin{array}{l}\infty \\
\infty \\
\dot{0} \\
\stackrel{\infty}{\sigma}\end{array}$ & $\begin{array}{l}\stackrel{9}{R} \\
\stackrel{0}{0} \\
0 \\
0\end{array}$ \\
\hline 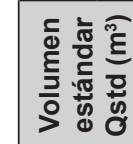 & 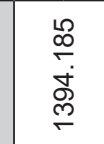 & 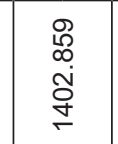 & 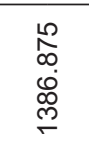 & $\stackrel{\stackrel{\stackrel{N}{N}}{N}}{\stackrel{N}{+}}$ & \begin{tabular}{l}
0 \\
$\vdots$ \\
$\dot{+}$ \\
$\dot{0}$ \\
\multirow{1}{*}{}
\end{tabular} & 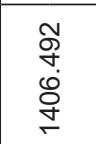 \\
\hline 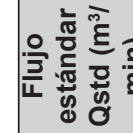 & $\stackrel{\widetilde{\Xi}}{\leftarrow}$ & $\stackrel{8}{\stackrel{8}{\leftarrow}}$ & 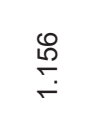 & 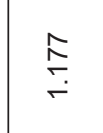 & $\stackrel{?}{\stackrel{R}{\leftarrow}}$ & 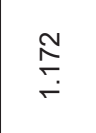 \\
\hline 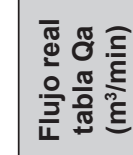 & $\stackrel{\Gamma}{\stackrel{5}{r}}$ & $\stackrel{\infty}{\stackrel{\infty}{\leftarrow}}$ & $\stackrel{8}{\stackrel{8}{ }}$ & 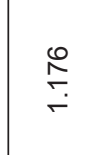 & 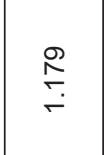 & 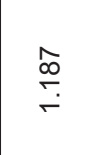 \\
\hline 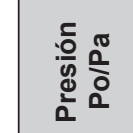 & $\begin{array}{l}\overline{\mathscr{g}} \\
0\end{array}$ & \begin{tabular}{l}
$\infty$ \\
$\&$ \\
\hdashline \\
0
\end{tabular} & $\begin{array}{l}\stackrel{8}{8} \\
\stackrel{8}{\circ} \\
0\end{array}$ & $\begin{array}{l}\mathscr{8} \\
\mathscr{8} \\
0\end{array}$ & $\begin{array}{l}\widetilde{\delta} \\
\stackrel{0}{\circ} \\
0\end{array}$ & 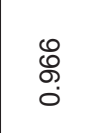 \\
\hline 늠 톨오 & 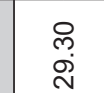 & $\begin{array}{l}\stackrel{8}{\infty} \\
\stackrel{\sim}{\sim}\end{array}$ & $\begin{array}{l}R \\
\dot{m}\end{array}$ & $\stackrel{\infty}{\infty}$ & 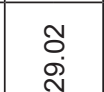 & 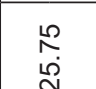 \\
\hline 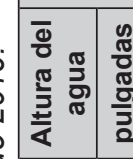 & 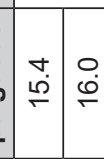 & 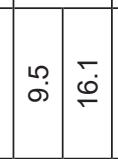 & \begin{tabular}{c|c}
$\bar{\phi}$ & $\infty$ \\
$\dot{\varphi}$ & $\stackrel{\oplus}{\varphi}$
\end{tabular} & \begin{tabular}{l|l}
$\mathfrak{f}$ \\
$\dot{f}$
\end{tabular} & 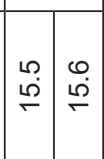 & 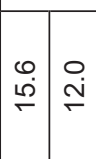 \\
\hline 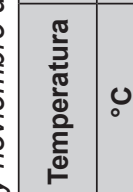 & $\begin{array}{ll}0 & 0 \\
\dot{N} & \stackrel{N}{N} \\
\text { N }\end{array}$ & 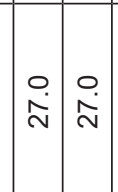 & \begin{tabular}{l|l}
$\circ$ & $\circ$ \\
$\stackrel{N}{N}$ & $\stackrel{\infty}{N}$
\end{tabular} & 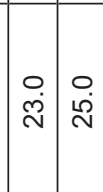 & 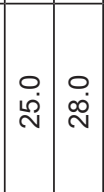 & \begin{tabular}{|c|c}
$\circ$ & 0 \\
$\stackrel{\infty}{j}$ & $\stackrel{\infty}{N}$ \\
$\sim$
\end{tabular} \\
\hline 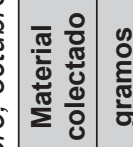 & 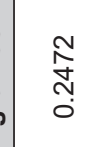 & ষ্ণ & $\begin{array}{l}\overline{8} \\
\varnothing \\
0\end{array}$ & 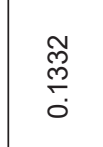 & ס్ల్ & 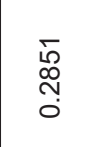 \\
\hline 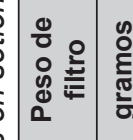 & 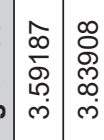 & 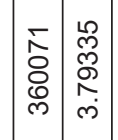 & 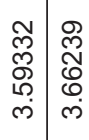 & 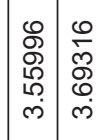 & 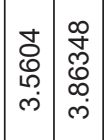 & 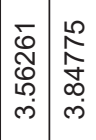 \\
\hline 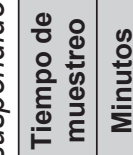 & ণ্ণ & ণ্ & 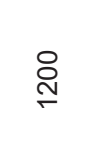 & 오 & ণ্ণ் & 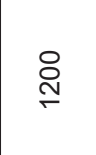 \\
\hline$\sum_{\Sigma}^{\infty}$ & & 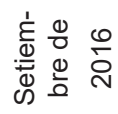 & & & 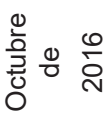 & \\
\hline$\stackrel{\circ}{\stackrel{ }{\underline{z}} \stackrel{\circ}{\mathrm{i}}}$ & 怘 & 闲 & $\stackrel{\circ}{m}$ & $\stackrel{ }{+}$ & $\stackrel{m}{\sigma}$ & $\stackrel{\circ}{\sigma}$ \\
\hline $\begin{array}{l}z z \\
\frac{z}{0} \\
\frac{0}{5} \\
w \\
w\end{array}$ & \multicolumn{6}{|c|}{ 岀 } \\
\hline
\end{tabular}


el fin de conocer los resultados en cuanto a la disminución de la polución ambiental en ese sector desde la perspectiva del trabajador de la planta.

Tabla 6. Disminución de la contaminación en el medio ambiente en área de chancado del sector beneficio San Nicolás de la empresa Shougang Hierro Perú S. A. A.

\begin{tabular}{|l|c|c|c|}
\hline Categorías & \multicolumn{3}{|c|}{ Rangos } \\
\hline Bajo & {$[0-2>$} & 2 & $13 \%$ \\
\hline Regular & {$[2-4>$} & 4 & $27 \%$ \\
\hline Alto & {$[4-6]$} & 9 & $60 \%$ \\
\hline TOTAL & 15 & $100 \%$ \\
\hline $\bar{X}$ & 5.27 & \\
\hline $\boldsymbol{S}$ & 2.07 & \\
\hline
\end{tabular}

Fuente: Elaboración propia a partir de los resultados obtenidos de la aplicación del cuestionario sobre la disminución de la polución ambiental.

\section{Interpretación}

Es importante mantener la revisión y seguimiento de fluctuación de los valores de la cantidad de polvo presente en el aire, de modo que no se alcancen los niveles críticos de $10 \mathrm{mg} / \mathrm{m}^{3}$ para la fracción de polvo inhalable y de $3 \mathrm{mg} / \mathrm{m}^{3}$ de polvo respirable (artículo 86 del Reglamento de Seguridad y Salud Ocupacional, 2012). En lo que respecta a los resultados mostrados en las Tablas 4, 5 y 6 de los polvos suspendidos producto del monitoreo durante el periodo 2016 , mediante el programa ambiental y usando el equipo para muestrear alto volumen High Vol PM10 (partículas con radio 5 micras o menos), estos arrojaron valores dentro del rango de 60 a $350 \mu \mathrm{g} / \mathrm{m}^{3}$ por día, con un promedio de $190 \mu \mathrm{g} / \mathrm{m}^{3}$ por día.

Los hallazgos contrastan que el sistema de mitigación de polvo de mineral de hierro es eficiente para la reducción del límite máximo permisible de calidad ambiental de $350 \mu \mathrm{g} / \mathrm{m}^{3}$ (dentro de las instalaciones de la empresa) y la exposición ocupacional a partículas de polvo respirable de $3 \mathrm{mg} / \mathrm{m}^{3}$ y $10 \mathrm{mg} / \mathrm{m}^{3}$ de polvo inhalable en el área de estudio. El valor de $350 \mu \mathrm{g} / \mathrm{m}^{3}$ puede ser encontrado e, inclusive, superado en la entrada de la planta donde se tratan los materiales particulados para la minimización de la cantidad de polvo.

En la Tabla 6, se observa que 2 trabajadores, que representan el $13 \%$ de la muestra de estudio, perciben que existe una baja disminución de la contaminación del medio ambiente en la zona en estudio de la citada empresa minera; 4 trabajadores, que representan el $27 \%$, perciben que existe una regular disminución de la contaminación del medio ambiente; y 9 trabajadores, que representan el $60 \%$, perciben que existe una alta disminución de la contaminación del medio ambiente.

Se determinó, a la vez, una media aritmética de 5.27 puntos, que señala una alta disminución de la contaminación en el área de estudio. Asimismo, se ha establecido una desviación estándar con una variación en \pm 2.07 con relación a la media aritmética, lo que demuestra que los datos son consistentes.

\section{CONCLUSIONES}

De acuerdo a los productos adquiridos de la indagación, se infirió que el sistema de mitigación de polvo de hierro es eficiente en lograr la minimización de la polución ambiental y exposición ocupacional.

1. Se ha determinado la eficacia del mencionado sistema.

2. Con el sistema implementado se ha reducido la exposición del personal en el interior del área de chancado al material particulado, sin exceder los límites del polvo respirable $\left(3 \mathrm{mg} / \mathrm{m}^{3}\right) \mathrm{ni}$ los límites del polvo inhalable $\left(10 \mathrm{mg} / \mathrm{m}^{3}\right)$.

3. Se ha verificado la reducción del límite máximo permisible para la calidad de aire de $500 \mu \mathrm{g} / \mathrm{m}^{3}$ en las instalaciones de la planta chancadora.

4. Se ha demostrado la posibilidad de reducción de registro de enfermedades ocupacionales en los trabajadores.

\section{AGRADECIMIENTOS}

A la Universidad Nacional Mayor de San Marcos y, en especial, a la Unidad de Posgrado de la Facultad de Ingenieria Geológica, Minera, Metalúrgica y Geográfica de dicha institución, por ofrecer oportunidades de superación profesional.

A nuestros asesores, el Dr. Oscar Tinoco Gomez y el Dr. Vidal Aramburú Rojas, por su apoyo y consejos en el desarrollo de esta investigación.

\section{REFERENCIAS BIBLIOGRÁFICAS}

[1] Aguilar, L. (2006). Contaminación ambiental. Recuperado de http://contaminacion-ambiente. blogspot.com.

[2] Anguita, F. y Moreno, F. (1993). Procesos geológicos externos y geología ambiental. Madrid, España: Rueda.

[3] Bartual, J. y Berenguer, M. J. (2001). NTP 607: Guías de calidad de aire interior: 
contaminantes químicos. Madrid, España: Instituto Nacional de Seguridad e Higiene en el Trabajo / Ministerio de Trabajo y Asuntos Sociales. Recuperado de https:// www.insst.es/documents/94886/326775/ ntp_607.pdf/0c6960b6-b461-4d21-9757e4ea03004327.

[4] Campos, I. (2000). Saneamiento ambiental. San José, Costa Rica: Editorial Universidad Estatal a Distancia.

[5] Castillo, H. (2008). Influencia antropogénica minera sobre la especie endémica Batrachophrynus macrostomus Peters, 1873 en peligro de extinción del lago Chinchaycocha (Pasco, Junín), 2007. (Tesis doctoral). Universidad Nacional de Trujillo, Perú. Recuperado de http://dspace.unitru.edu.pe/ bitstream/handle/UNITRU/5903/Tesis\%20 Doctorado\%20-\%20HItlser\%20Castillo\%20 Paredes.pdf?sequence=1\&isAllowed=y.

[6] García, A. y Fernández, R. (6 de diciembre de 2008). Decreto Supremo n. $^{\circ}$ 007-2008-MINAM. Aprueban Reglamento de Organización y Funciones del Ministerio del Ambiente. Recuperado de http:// www.minam.gob.pe/wp-content/uploads/2013/09/ ds_007-2008-minam.pdf.

[7] Glave, M. y Kuramoto, J. (2002). Minería, minerales y desarrollo sustentable en Perú. En Equipo MMSD América del Sur, Minería, minerales y desarrollo sustentable en América del Sur (pp. 529-591). Londres, Reino Unido: Centro de Investigación y Planificación del Medio Ambiente / Centro Internacional de Investigación para el Desarrollo / Iniciativa de Investigación sobre Políticas Mineras.

[8] Herrera, P. y Millones, O. (2011). ¿Cuál es el costo de la contaminación ambiental minera sobre los recursos hídricos en el Perú? Informe final. Lima, Perú: Consorcio de Investigación Económica y Social / Departamento de Economía de la Pontificia Universidad Católica del Perú. Recuperado de http://www2.congreso. gob.pe/sicr/cendocbib/con3_uibd.nsf/FF 88A00731054C670525797A006117B0/\$FILE/ Informe_Final_Costo_Economico_de_la_ contaminacion_en_los_recursos_Hidrico.pdf.

[9] Hokama, D. (16 de julio de 1996). Resolución Ministerial n.o 315-96 EM/VMM. Niveles máximos permisibles de elementos $y$ compuestos presentes en emisiones gaseosas provenientes de las unidades minero-metalúrgicas. Recuperado de http:// intranet2.minem.gob.pe/web/archivos/dgaam/ publicaciones/compendio99/rm315-96.pdf.

[10] Humala, O. y Villena, P. (20 de agosto de 2011). Reglamento de la leyn. ${ }^{\circ} 29783$, Ley de Seguridad y Salud en el Trabajo. Decreto Supremo n. ${ }^{\circ} 005-$ 2012-TR. Recuperado de https://www.minagri. gob.pe/portal/download/pdf/seguridad-y-salud/ reglamento-ley29783.pdf.

[11] Humala, O. y Villena, J. (25 de abril de 2012). Decreto Supremon. ${ }^{\circ} 005-2012-T R$. Reglamento de la Leyn. ${ }^{\circ} 29783$, Ley de Seguridad y Salud en el Trabajo. Recuperado de https://busquedas. elperuano.pe/normaslegales/reglamento-dela-ley-n-29783-ley-de-seguridad-y-salud-en-edecreto-supremo-n-005-2012-tr-781249-1/.

[12] Martínez, C., Quero, A., Isidro, J. y Rego, G. (2001). Enfermedades pulmonares profesionales por inhalación de polvos inorgánicos. Jano, 61(1397), 34-39.

[13] Medina, F. (2008). Inventario de pasivos ambientales mineros. Lima, Perú: Proyecto de Reforma del Sector de Recursos Mineros del Perú.

[14] Ministerio de Energía y Minas (10 de diciembre de 1993). Reglamento sobre protección del medio ambiente. Decreto Supremo n. ${ }^{\circ}$ 059-93-EM. Recuperado de http://www.minem.gob.pe/_legislacionM. php?idSector=4\&idLegislacion=5272.

[15] Moraga, P., Rivera, L. y Soto, M. (2013). Evaluar y proponer la disminución de material particulado en la planta de chancado secundario-terciario en división El Teniente de Codelco Chile. (Tesis de grado). Universidad Academia de Humanismo Cristiano, Chile. Recuperado de http://bibliotecadigital. academia.cl/handle/123456789/1236.

[16] Organismo de Evaluación y Fiscalización Ambiental (2014). Cartilla del abc de la fiscalización ambiental. Lima, Perú: Organismo de Evaluación y Fiscalización Ambiental. Recuperado de https://centroderecursos. cultura.pe/sites/default/files/rb/pdf/ Brochure $\% 20$ EI\%20ABC $\% 20$ de $\% 201$ a $\% 20$ fiscalizacion\%20ambiental.pdf.

[17] Vega, D. (2007). El origen de polvos y su mitigación en la minería. Trabajo de investigación. Monografias.com. Recuperado de https://www.monografias.com/trabajos53/ contaminacion-polvos-mineria/contaminacionpolvos-mineria.shtml.

[18] Warner, P. (1981). Análisis de los contaminantes del aire. Madrid, España: Paraninfo. 


\section{Iron Ore Dust Control System at the San Nicolás-Marcona Benefit Concession Crushing Plant}

CARlos YABAR Baños ${ }^{1}$ Vidal Aramburú Rojas ${ }^{2}$ FÉlix TiNOCO ÁNGELES ${ }^{3}$

\begin{abstract}
The objective of this applied research is to demonstrate the decrease in environmental pollution and occupational exposure by systematizing the iron ore dust control process at the San Nicolás benefit concession crushing plant of mining company Shougang Hierro Perú S. A. A. The causal relationship between environmental pollution and occupational exposure was established via the measurement of particulate matter. Perception of environmental pollution reduction was measured using a quantitative approach in a sample of 15 workers and data processing by means of descriptive and inferential statistical methods. The results show that the average level of influence of the iron ore dust control system is significant, at a confidence level of $95 \%$ and significance level of $5 \%$. Therefore, the system efficiently reduces air pollutant values. Prior to implementation, the air pollutant value was $500 \mu \mathrm{g} / \mathrm{m}^{3}$, above the maximum permissible limit established in the current environmental quality standards; the occupational exposure to respirable dust particles was $3.2 \mathrm{mg} / \mathrm{m}^{3}$, and inhalable dust particles values, $13 \mathrm{mg} / \mathrm{m}^{3}$. After conducting environmental and occupational monitoring, air pollutant values of less than $220 \mu \mathrm{g} / \mathrm{m}^{3}$ were obtained, and occupational exposure to respirable dust particles and inhalable dust was reduced to $2.2 \mathrm{mg} / \mathrm{m}^{3}$ and $9.2 \mathrm{mg} / \mathrm{m}^{3}$, respectively, at the San Nicolás benefit concession crushing plant. It is concluded that the dust control system efficiently minimizes iron ore dust in the mentioned plant.
\end{abstract}

Keywords: control; dust; crushing plant; occupational health; occupational safety.

\section{INTRODUCTION}

Mining is generally considered harmful and damaging for the environment, as it leads to poor health in communities. Currently, technological development allows the use of resources to minimize this situation, as a result of the demands of the population and the globalized world, in which cleaner progress is sought.

This study was conducted at the San Nicolás de Shougang Hierro Perú S. A. A. benefit concession crushing plant, where the presence of particulate matter was observed. In light of this situation, it was necessary to reduce the amount of molecules present in the environment (especially in the vicinity of the plant) by installing a system that absorbs this particulate cloud.

The references used for this task were Supreme Decree No. 007-2008-MINAM (García \& Fernández, December 6, 2008), environmental monitoring standards set forth in Supreme Decree No. 059-93-EM (Ministerio de Energía y Minas, December 10, 1993), and the new Ley de Seguridad y Salud en el Trabajo N. ${ }^{\circ}$ 29783 [Occupational Safety and Health Law No. 29783] enacted in 2011(Humala \& Villena, August 20, 2011; Humala \&Villena, April 25, 2012).

Particulates result from different mining operations, in which a series of dust emissions are released into the atmosphere; predominantly, particulates are generated during mineral extraction activities such as blasting, loading, hauling, primary or secondary crushing of ore and belt conveyor transportation. The monitoring of $\mathrm{PM}_{10}$ concentrations registered daily arithmetic means higher than $350 \mu \mathrm{g} / \mathrm{m}^{3}$, the maximum permissible limit approved by Ministerial

1 Materials engineer from the Universidad Nacional San Agustín de Arequipa (Arequipa, Peru). Currently working as loss prevention specialist at Shougang Hierro Perú S. A. A. (Ica, Peru) ORCID: https://orcid.org/0000-0003-0854-6852

E-mail: cyabar@shp.pe

$2 \mathrm{PhD}$ in Business Management and MSc in Metallurgical Engineering from the Universidad Nacional Mayor de San Marcos (Lima, Peru). Currently working as professor at the School of Geological, Mining, Metallurgical and Geographical Engineering of the UNMSM (Lima, Peru). ORCID: 0000-0001-7411-3866

E-mail: varamburu@unmsm.edu.pe

3 Master degree in Logistics and Operations Management from the Universidad Nacional Mayor de San Marcos (Lima, Peru).Currently working as an independent consultant.

ORCID: https://orcid.org/0000-0002-2630-4657

E-mail: tinocofelix@gmail.com 
Resolution No. 315-96 EM/VMM (Hokama, July 16, 1996) (see Table 1).

An environmental aspect of special consideration is the wind speed in the district of San Juan de Marcona, Nazca Province, Ica Department, considered as one with locations with the highest wind rates in the country, with an average of $9 \mathrm{~m} / \mathrm{s}$ at 40 MASL. (Glave \& Kuramoto, 2002). This is an important factor to consider in the process of iron dust control at the secondary crushing plant, located at 3200 MASL.

\section{Pathological effects of dust from mining activities}

For Martínez, Quero, Isidro and Rego (2001), in principle, these particulate clouds reduce ambient light due to their composition, which in turn has a detrimental impact on health. This occurs because of the size of particles and the combination of several elements. Particles are variable in size, which allows them to stay in the air due to the lift force; particles with smaller size and density are carried for longer periods and distances. Once wind speed drops, particles tend to fall to the ground.

Low density particles smaller than $2.3 \mathrm{mg} / \mathrm{m}^{3}$ remain in the air for a relatively longer time, making it possible for them to be inhaled by living organisms, negatively impacting their health. When particle concentration values are below $10 \mathrm{mg} / \mathrm{m}^{3}$, they are more easily and continuously absorbed and have the potential to cause major health problems.

The maximum permissible concentration levels for suspended particulate matter is $350 \mu \mathrm{g} / \mathrm{m}^{3}$, as set forth in the National Air Quality Standards for air preservation. The arithmetic mean determined that daily concentration levels of particulate matter $\left(P_{10}\right)$ exceed the established limits, which demonstrates the need for control measures to minimize pollution.

\section{Selection and procurement of equipment to reduce pollutants}

According to studies conducted by Warner (1981), and Anguita and Moreno (1993), spacing between filters is reduced in order to capture very small particles contained in the air. The collection of suspended particulate matter will depend directly on their size and the subsequent use of a process incorporating the dust mitigation system (see Table 2).

Further to the above, Vega (2007) indicates that the selection of equipment varies depending on the retention of pollutant particles. Information regarding particles' physical characteristics in terms of size, shape or any other specific features is not enough to determine equipment selection, as the particles' chemical composition is more important and determines their impact on health.

\section{Dust collection system}

The research by Moraga, Rivera and Soto (2013), conducted in Codelco, Chile, addresses the case of a secondary and tertiary crushing plant; in addition, it describes 19 operating dust collectors, of which 13 are Aeromix Wet Scrubbers and 6 are Rotoclone wet dust collectors. These dust removal devices are positioned in places where they can continuously and permanently absorb particles from belt conveyors that transport the crushed material. Captured dust is then mixed with water so as to agglomerate it to be poured into the drainage channels, from where, separated from water, the material is collected. This accumulation of minerals is recovered for processing.

In general, this group of collectors is in continuous operation; accordingly, a maintenance plan should be in place to avoid unwanted shutdowns that may affect dust particle collection and may cause largescale pollution. In turn, qualified staff should keep a

Table 1. Maximum permissible limits established for air quality.

\begin{tabular}{|l|c|c|c|}
\hline Parameter & $\begin{array}{c}\text { Arithmetic mean daily } \\
\text { concentration } \\
\mu \mathrm{g} / \mathbf{m}^{3}(\mathbf{p p m})\end{array}$ & $\begin{array}{c}\text { Arithmetic mean annual } \\
\text { concentration } \\
\boldsymbol{\mu g} / \mathrm{m}^{3}\end{array}$ & $\begin{array}{c}\text { Geometric mean annual } \\
\text { concentration } \\
\boldsymbol{\mu g} / \mathbf{m}^{3}\end{array}$ \\
\hline Sulphur dioxide & $572^{*}$ & 172 & - \\
\hline Particulate matter & $350^{*}$ & - & 150 \\
\hline Lead & - & 0.5 & - \\
\hline Arsenic & 6 & - & - \\
\hline
\end{tabular}

* Should not be exceeded more than once a year.

Source: Hokama (July 16, 1996). 
Table 2. Captured particle size range.

\begin{tabular}{|l|c|}
\hline Equipment & Captured particle size range in microns \\
\hline $\begin{array}{l}\text { Electrostatic precipitator } \\
\text { Packed columns }\end{array}$ & 0.01 to 90 \\
\hline Paper filters & 0.01 to 100 \\
\hline Fabric filters & 0.005 to 8 \\
\hline Gas scrubbers & 0.05 to 90 \\
\hline Centrifugal separator & 0.05 to 100 \\
\hline Sedimentation chambers & 5 to 100 \\
\hline
\end{tabular}

Source: Vega (2007).

record on the use of the equipment (Moraga et al., 2013).

The physical and chemical properties of particles are invariable; however, their size, shape and specific features are subject to the amount of moisture present in the material, and to the equipment and its maintenance. It is important to consider the elevation of the accumulated material, as well as weather, such as precipitation levels, wind direction and wind speed variability.

It is important to note that Martínez et al. (2001) mention size variability. Depending on size, particles are lodged in the lung system and deteriorate workers' health. The largest particles lodge in the trachea, nasal cavity and bronchial tubes; medium-size particles, in the lung area; and extra-fine particles, in alveoli.

\section{Permissible levels of pollutants in air}

Current levels of permissible air pollution, used as reference, are those unlikely to generate health problems. These values are set forth in the Peruvian Environmental Quality Standards (EQA) for air, established by the Ministerio del Ambiente. These values should be regarded as primary standards essential in the development of environmental policies, mining projects, and other project types.

Similarly, the United States Environmental Protection Agency (EPA) establishes air pollution limits; primary standards for children, asthmatics, and the elderly; for protection against decreased visibility, as well as damage to flora and fauna (Bartual \& Berenguer, 2001).

\section{Environmental pollution}

According to Aguilar (2006), environmental damage is the result of various agents present in air, water and areas where living conditions are threatened by toxic elements, whether biochemical, biophysical, or simply physical or chemical; furthermore, one or all of them may occur at the same time. Either way, all these elements affect human development and increase degradation of the flora and fauna. Campos (2000) adds to this definition noting that the existence of biotic factors, such as living organisms and organic substances, and non-biotics factors, such as water, air and minerals, all affect the environment in some way.

\section{Mining environmental liabilities}

Herrera and Millones (2011) state that water consumption is a factor that constantly disrupts agricultural development in the areas near crushing plants; this disruption is linked to the washing of large quantities of extracted material. This relatively contaminated water affects the Locumba, Cañete, Mantaro, Acarí and Moche hydrographic basins. In addition, a portion of these contaminated waters reaches the Pacific Ocean. In the case of the Mantaro River, Medina (2008) observes that the consumption of water used in the washing of extracted material has caused animals and farming land losses, and has significantly damaged the economy of the area. The company involved in said damages states that, at present, the river only acts as a drainage channel for nearby plants. In like manner, the ecological damage caused to the Reserva Nacional de Junín [Junin National Reserve] at Lake Chinchaycocha, must be mentioned, as it has contributed to the disappearance of animals, rivers and plants, and to the deterioration in air quality. It has also caused the disappearance of endemic species such as the Junin grebe and other birds (Castillo, 2008). 


\section{METHODOLOGY}

\section{Implementation of ore dust control system at crushing plant No. 1-San Nicolás}

Ecosystem monitoring is aimed at determining the presence and concentration of pollutants in the environment; it also enables the assessment of the state of natural resources (Organismo de Evaluación y Fiscalización Ambiental, 2014).

General conditions were: elevation of 83.5 MASL, maximum air temperature of $32{ }^{\circ} \mathrm{C}$, minimum air temperature of $18{ }^{\circ} \mathrm{C}, 95 \%$ relative humidity, and saline atmosphere (see Figure 1).

\section{Description of the project}

Implementation was as follows:

- Installation of dust collectors, collection hoods and metal supports from line 1 and vibrating sieve.

- Assembly of electrostatic precipitator.

- Assembly of fine-particle dust conveyors.

- Assembly of fan.

- Assembly of chimney.

- Construction of ponds.

- Assembly and setting up of compressor.
- Assembly and setting up of $3 \mathrm{~m}^{3}$ air receiver tank.

- Assembly of injection line for filter cleaning.

- Installation of boot and control systems and auxiliary equipment.

- Assembly and installation of medium voltage electrical equipment (4.16 kV cells and/or starters) and low voltage electrical equipment (440 V, $220 \mathrm{~V}, 120 \mathrm{~V})$.

- Assembly and installation of electrical infrastructure and ducting systems for the operation and supervision of different electromechanical equipment.

- Installation of electric power system infrastructure, instrumentation, automation and monitoring in general.

- Construction of fully equipped electrical substation.

Table 3 shows the technical parameters considered by the company for the implementation of the system.

\section{RESULTS AND DISCUSSION}

A sample of 15 workers exposed to dust or particulate matter produced at the secondary crushing plants was analyzed. Staff working in plant have also been monitored, including mechanical and

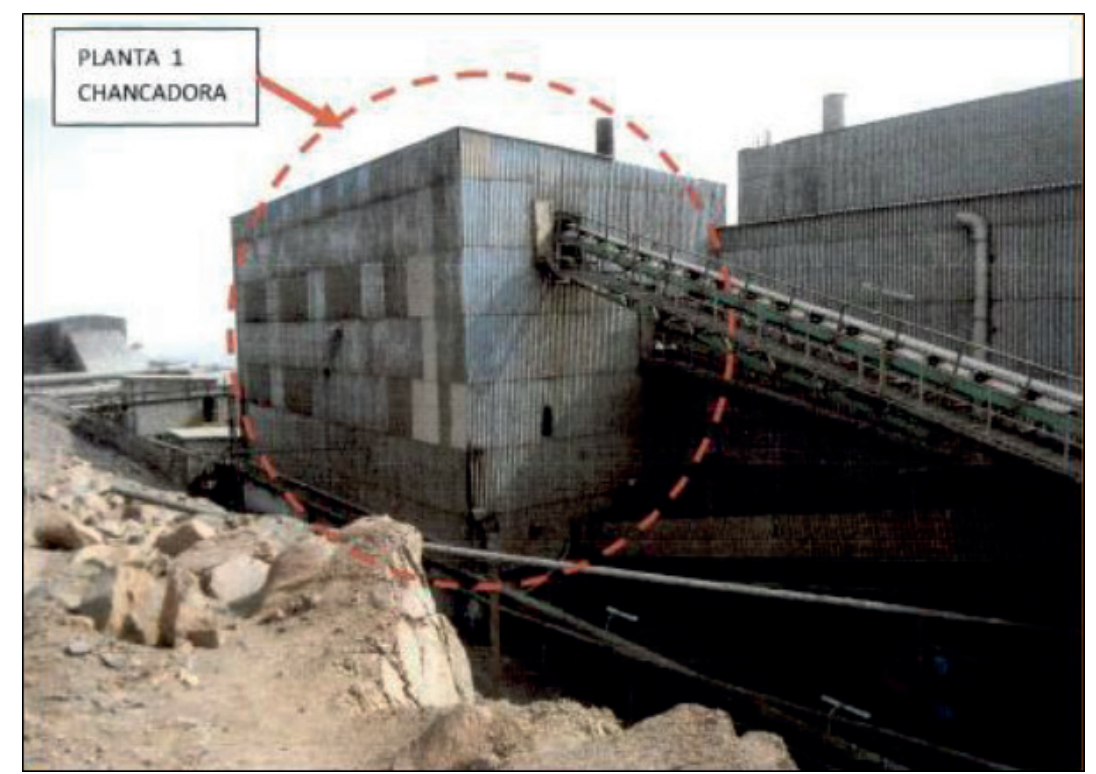

Figure 1. Shougang Hierro Perú S. A. A. crushing plant Source: Prepared by the authors. Photo by Carlos Yabar Baños. 
Table 3. Technical parameters. Shougang Hierro Perú S. A. A.

\begin{tabular}{|l|c|}
\hline \multicolumn{1}{|c|}{ Parameter } & Unit \\
\hline Processed air volume & $450000 \mathrm{~m}^{3} / \mathrm{h}$ \\
\hline Filtering area & $7200 \mathrm{~m}^{2}$ \\
\hline Wind speed in filter & $1.04 \mathrm{~m} / \mathrm{min}$ \\
\hline Equipment's resistance & $1800 \mathrm{~Pa}$ \\
\hline Number of filter chambers & 16 \\
\hline Number of filter bags & 2400 \\
\hline Dimensions of filter bags & $0160 \times 6000 \mathrm{~mm}$ \\
\hline Dimensions of filter bags support cages & $0155 \times 5980 \mathrm{~mm}$ \\
\hline Number of filter bags support cages & 2400 \\
\hline Material of filters & 160 \\
\hline Number of pulse valves & $3 "$ \\
\hline Dimensions of pulse valves & $300 \times 300$ \\
\hline Dimensions of flashboard valves & 8 \\
\hline Number of discharge valves & YJD-16A \\
\hline Type of discharge valves & 16 \\
\hline Number of off-line valves & Polyester Needle Felt \\
\hline
\end{tabular}

Source: Prepared by the authors

maintenance staff members, and equipment operators. The equipment used for the monthly monitoring of $\mathrm{PM}_{10}$ was set up in strategic locations approved by the Programa de Adecuación y Manejo Ambiental ${ }^{\mathrm{TN} 1}$ (PAMA).

TN1: Program that established a set of projects, agreed with the Peruvian government, with the purpose to reduce the environmental impact of mining activities.

Sample selection was done gradually; workers with greater exposure to pollutants were first selected, and, then, those with less exposure. Each monitoring session was recorded to be subsequently compared against prior records.

Environmental monitoring of $\mathbf{P M}_{10}$. Its purpose is to assess tiny dust-in solid or liquid-ash, soot, metal and cement particles, which are dispersed in atmospheric space and have a radius of less than $5 \mu \mathrm{m}$. They are composed of inorganic substances, such as aluminum and silica salts, heavy metals, among others. Organic particulate is associated with carbon dust.

In-person respirable and inhalable dust monitoring using pump Escort Elf MSA. The study was conducted to analyze results using the NIOSH sampling methodology in order to determine respirable dust concentrations in the workplace (via method 0600, which measures not otherwise regulated respirable particles).

Weather station. The device for regularly measuring and detecting variable atmospheric conditions. The data obtained are used for weather prediction, based on numerical models, and for climate research.

Risk maps. The crushing plant's map indicating warning, informative, mandatory and fire safety signs.

HIRAC matrix. The matrix used for hazard identification, risk assessment, and control measures.

Tables 4 and 5 show the results of dust particulate matter monitoring between June and October 2016 conducted using a High-Vol $\mathrm{PM}_{10}$ sampler.

The results obtained in the post-test evaluation upon measuring environmental pollution are illustrated below. A questionnaire was applied to 15 workers at the San Nicolás crushing plant of Shougang Hierro Perú S. A. A. (see Table 6), in order to understand the results in terms of pollution reduction in this area from the plant worker's perspective. 


\begin{tabular}{|c|c|c|c|c|c|c|}
\hline है & స్ల్ & 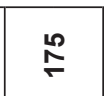 & $\underset{N}{\mathbb{Z}}$ & 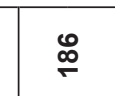 & $\stackrel{\mathscr{N}}{\sim}$ & ๑ \\
\hline 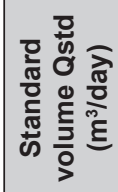 & $\begin{array}{l}\hat{0} \\
\stackrel{0}{0} \\
\stackrel{5}{r}\end{array}$ & $\begin{array}{l}\stackrel{m}{S} \\
\stackrel{N}{N} \\
\stackrel{N}{N}\end{array}$ & 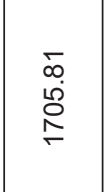 & $\begin{array}{l}\stackrel{0}{m} \\
\stackrel{N}{N} \\
\stackrel{5}{\leftarrow}\end{array}$ & 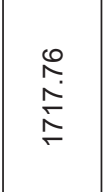 & $\begin{array}{l}\stackrel{0}{0} \\
\stackrel{N}{N} \\
\stackrel{5}{\leftarrow}\end{array}$ \\
\hline 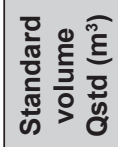 & $\begin{array}{l}\underset{N}{\mathbb{N}} \\
\stackrel{\sim}{\sim}\end{array}$ & 兽 & 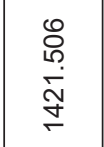 & 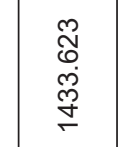 & 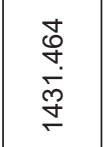 & 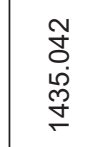 \\
\hline 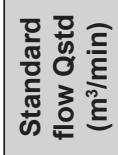 & $\stackrel{\infty}{\leftarrow}$ & 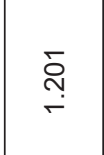 & $\stackrel{\infty}{\leftarrow}$ & $\stackrel{\stackrel{\circ}{\circ}}{\leftarrow}$ & 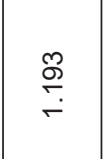 & $\stackrel{\mathscr{8}}{\stackrel{一}{\leftarrow}}$ \\
\hline 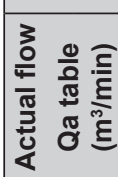 & $\stackrel{\infty}{\stackrel{\infty}{\leftarrow}}$ & $\stackrel{\infty}{\stackrel{\infty}{\leftarrow}}$ & $\stackrel{\infty}{\stackrel{\infty}{\leftarrow}}$ & $\stackrel{\infty}{\leftarrow}$ & $\stackrel{\infty}{\stackrel{\infty}{\leftarrow}}$ & $\stackrel{\stackrel{\circ}{\circ}}{\leftarrow}$ \\
\hline 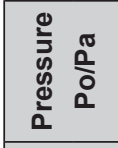 & \begin{tabular}{l}
\multirow{O}{\circ}{} \\
$\stackrel{\circ}{0}$
\end{tabular} & $\begin{array}{l}\text { Na } \\
\hat{\sigma} \\
0\end{array}$ & $\begin{array}{l}\widetilde{O} \\
\stackrel{0}{\circ} \\
0\end{array}$ & 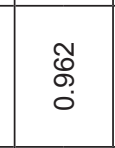 & 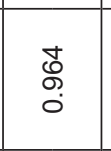 & $\begin{array}{l}\mathscr{8} \\
\stackrel{8}{0} \\
0\end{array}$ \\
\hline$\underset{\underline{\xi}}{E}$ 옴 & 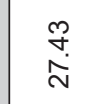 & $\frac{8}{\dot{亠}}$ & 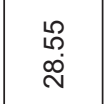 & $\begin{array}{l}\infty \\
\infty \\
\infty \\
\stackrel{\infty}{0}\end{array}$ & $\stackrel{\stackrel{n}{n}}{\stackrel{N}{\sim}}$ & $\bar{\emptyset}$ \\
\hline 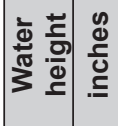 & 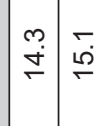 & $\stackrel{\circ}{\stackrel{\circ}{F}} \stackrel{\stackrel{\leftrightarrow}{F}}{\stackrel{P}{F}}$ & 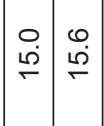 & 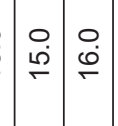 & 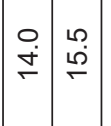 & $\stackrel{\stackrel{m}{\dot{M}}}{\stackrel{\rho}{\stackrel{m}{r}}}$ \\
\hline 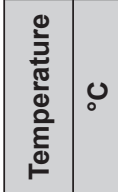 & $\begin{array}{l}\circ \\
\stackrel{i}{N} \\
\stackrel{\sim}{~}\end{array}$ & \begin{tabular}{l|l}
0 & 0 \\
$\stackrel{N}{N}$ & $\stackrel{N}{N}$ \\
\end{tabular} & \begin{tabular}{|c|c|}
$\stackrel{0}{\text { N }}$ & 0 \\
$\stackrel{N}{N}$
\end{tabular} & \begin{tabular}{c|c|}
$\circ$ & 0 \\
$\grave{\sim}$ & $\stackrel{\dot{\nu}}{N}$ \\
\end{tabular} & \begin{tabular}{l|l}
$\circ$ & 0 \\
$\dot{\sim}$ & $\stackrel{2}{\stackrel{N}{N}}$
\end{tabular} & \begin{tabular}{l|l}
$\circ$ & 0 \\
$\stackrel{\sim}{~}$ & $\stackrel{\sim}{N}$
\end{tabular} \\
\hline 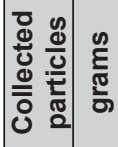 & 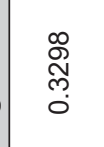 & 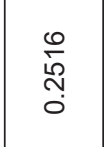 & $\begin{array}{l}8 \\
\stackrel{0}{0} \\
\text { ల్ } \\
0\end{array}$ & $\begin{array}{l}\text { R } \\
0 \\
0 \\
0\end{array}$ & $\begin{array}{l}\mathscr{Q} \\
\stackrel{8}{0} \\
\vdots\end{array}$ & 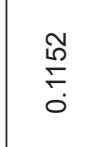 \\
\hline 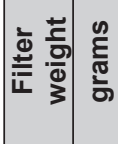 & 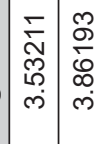 & 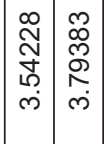 & 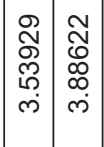 & 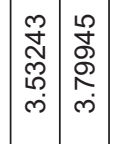 & 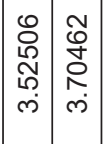 & 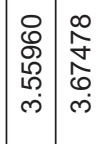 \\
\hline 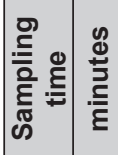 & 옳 & ণ্ণ & ণ্ণ & ণ & ণ & 옳 \\
\hline 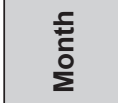 & & $\stackrel{\oplus}{\stackrel{0}{g}} \stackrel{0}{\stackrel{N}{N}}$ & & & 촉 & \\
\hline 产 & $\stackrel{\circ}{\circ}$ & $\stackrel{N}{N}$ & $\stackrel{N}{N}$ & $\stackrel{\infty}{N}$ & ষ্ল & ల్ల \\
\hline $\begin{array}{l}z \\
\frac{\partial}{E} \\
\frac{1}{5} \\
\text { en }\end{array}$ & \multicolumn{6}{|c|}{ 点 } \\
\hline
\end{tabular}

\begin{tabular}{|c|c|c|c|c|c|c|}
\hline$\stackrel{\text { है }}{\text { के }}$ & $\stackrel{શ}{F}$ & $\stackrel{\mathscr{P}}{\stackrel{2}{2}}$ & in & ১ & $\stackrel{0}{N}$ & ని \\
\hline 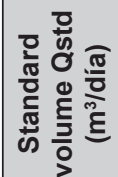 & $\begin{array}{l}\tilde{N} \\
\stackrel{0}{0} \\
\stackrel{0}{0}\end{array}$ & 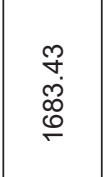 & 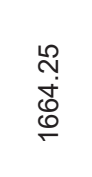 & $\begin{array}{l}\hat{0} \\
\dot{0} \\
0 \\
0\end{array}$ & 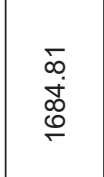 & $\begin{array}{l}\stackrel{9}{2} \\
\stackrel{0}{\infty} \\
\stackrel{0}{0}\end{array}$ \\
\hline 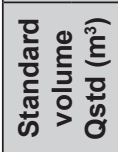 & $\begin{array}{l}\stackrel{\infty}{\infty} \\
\stackrel{\infty}{\dot{m}} \\
\stackrel{0}{\square}\end{array}$ & 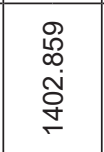 & 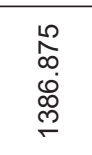 & $\begin{array}{l}\stackrel{\stackrel{N}{N}}{N} \\
\stackrel{N}{+}\end{array}$ & $\begin{array}{l}0 \\
\vdots \\
\dot{0} \\
\text { ㅁ }\end{array}$ & 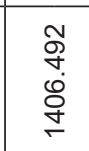 \\
\hline 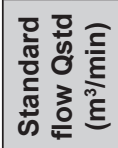 & 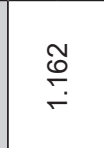 & $\stackrel{8}{\stackrel{8}{\leftarrow}}$ & $\stackrel{\circ}{\stackrel{0}{?}}$ & $\stackrel{尺}{\stackrel{F}{\leftarrow}}$ & $\stackrel{?}{\stackrel{R}{\leftarrow}}$ & 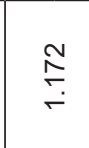 \\
\hline 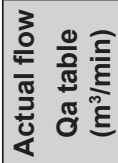 & $\stackrel{\Gamma}{\stackrel{5}{r}}$ & $\stackrel{\infty}{\stackrel{\infty}{\leftarrow}}$ & $\stackrel{8}{\stackrel{\circ}{\leftarrow}}$ & $\stackrel{0}{\stackrel{2}{\leftarrow}}$ & 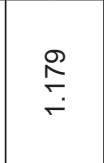 & $\stackrel{\infty}{\stackrel{\infty}{\leftarrow}}$ \\
\hline 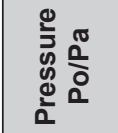 & $\begin{array}{l}\bar{\delta} \\
\varnothing \\
\end{array}$ & $\begin{array}{l}\infty \\
\stackrel{\infty}{0} \\
\stackrel{0}{0}\end{array}$ & 惫 & $\begin{array}{l}\mathscr{0} \\
\mathscr{0} \\
0\end{array}$ & 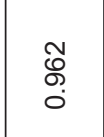 & $\begin{array}{l}\mathscr{8} \\
\% \\
0\end{array}$ \\
\hline 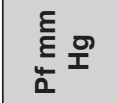 & $\begin{array}{l}\stackrel{0}{\text { }} \\
\text { iे }\end{array}$ & $\begin{array}{l}\stackrel{8}{\infty} \\
\stackrel{\sim}{\infty}\end{array}$ & $\begin{array}{l}R \\
\dot{m} \\
\dot{m}\end{array}$ & $\stackrel{\infty}{\infty}$ & 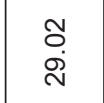 & $\stackrel{\stackrel{\rho}{R}}{\stackrel{\sim}{\sim}}$ \\
\hline 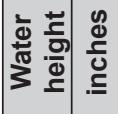 & 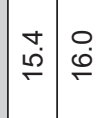 & 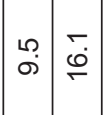 & 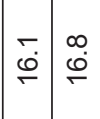 & 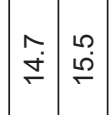 & \begin{tabular}{l|l}
$\stackrel{0}{0}$ & 0 \\
$\stackrel{\rho}{\circ}$ & $\stackrel{\rho}{\varphi}$
\end{tabular} & 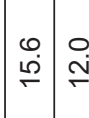 \\
\hline 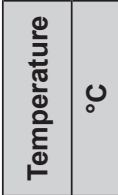 & \begin{tabular}{l|l}
$\stackrel{0}{0}$ & $\stackrel{\leftrightarrow}{\sim}$ \\
$\stackrel{N}{N}$
\end{tabular} & \begin{tabular}{ll|}
$\stackrel{0}{i}$ & 0 \\
$\stackrel{N}{N}$
\end{tabular} & \begin{tabular}{l|l}
0 & 0 \\
$\stackrel{i}{N}$ & $\stackrel{\infty}{N}$ \\
\end{tabular} & 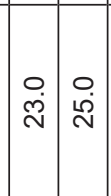 & 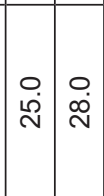 & \begin{tabular}{|l|l}
0 & 0 \\
$\sim$ & $\infty$ \\
$\sim$ & $\infty$ \\
$\sim$
\end{tabular} \\
\hline 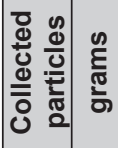 & 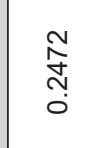 & $\begin{array}{l}\stackrel{8}{0} \\
\text { ஸ̦ } \\
0\end{array}$ & $\begin{array}{l}\overline{8} \\
\varnothing \\
0\end{array}$ & 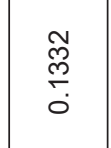 & $\begin{array}{c}\bar{\delta} \\
\text { } \\
0\end{array}$ & 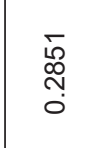 \\
\hline 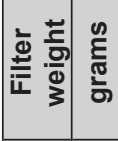 & 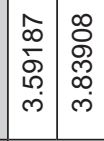 & 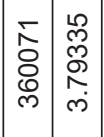 & 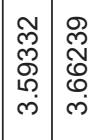 & 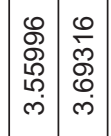 & 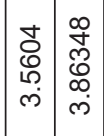 & 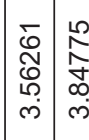 \\
\hline 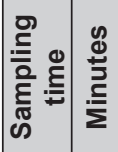 & 옳 & ণ্ণ & 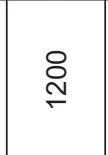 & 옴 & 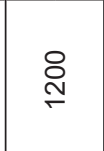 & 옳 \\
\hline $\begin{array}{l}\overline{\underline{E}} \\
\bar{\partial} \\
\Sigma\end{array}$ & \multicolumn{3}{|c|}{ 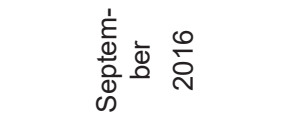 } & \multicolumn{3}{|c|}{ 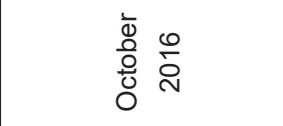 } \\
\hline 产 & $\begin{array}{l}\stackrel{\leftrightarrow}{\circ} \\
\text { D. }\end{array}$ & న్ల & 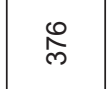 & $\frac{}{\dot{\gamma}}$ & $\stackrel{m}{\frac{m}{\sigma}}$ & $\stackrel{0}{\dot{\sigma}}$ \\
\hline$\frac{z}{\frac{2}{E}}$ & \multicolumn{6}{|c|}{$\hat{\dot{山}}$} \\
\hline
\end{tabular}


Table 6. Pollution reduction at the Shougang Hierro Perú S. A. A. San Nicolás benefit concession crushing plant.

\begin{tabular}{|l|c|c|c|}
\hline Categories & \multicolumn{3}{|c|}{ Ranges } \\
\hline Low & {$[0-2>$} & 2 & $13 \%$ \\
\hline Normal & {$[2-4>$} & 4 & $27 \%$ \\
\hline High & {$[4-6]$} & 9 & $60 \%$ \\
\hline TOTAL & 15 & $100 \%$ \\
\hline $\bar{X}$ & & 5.27 & \\
\hline $\boldsymbol{S}$ & 2.07 & \\
\hline
\end{tabular}

Source: Prepared by the authors based on the results obtained from the questionnaire on environmental pollution.

\section{Interpretation}

It is important to continue monitoring the fluctuation of dust concentrations in the air, in order to prevent respirable and inhalable dust from reaching critical values of $10 \mathrm{mg} / \mathrm{m}^{3}$ and $3 \mathrm{mg} / \mathrm{m}^{3}$, respectively (Artículo 86 del Reglamento de Seguridad y Salud Ocupacional, 2012). The values shown in Tables 4, 5 and 6 for suspended particle matter obtained from the monitoring conducted during 2016, by means of the environmental program and using the High Vol $\mathrm{PM}_{10}$ sampler (particles with radius of 5 microns or less), are within the range of 60 to $350 \mu \mathrm{g} / \mathrm{m}^{3}$ per day, with an average of $190 \mu \mathrm{g} / \mathrm{m}^{3}$ per day.

The findings corroborate that the iron ore dust control system can efficiently reduce air pollutant concentrations, within the company's facilities, to the maximum permissible limit of environmental quality of $350 \mu \mathrm{g} / \mathrm{m}^{3}$, as well as occupational exposure to respirable and inhalable dust particles of $3 \mathrm{mg} / \mathrm{m}^{3}$ and $10 \mathrm{mg} / \mathrm{m}^{3}$, respectively. Values of $350 \mu \mathrm{g} / \mathrm{m} 3$ or even lower values can be achieved at the entrance of the plant where particulate matter is treated to minimize the amount of dust.

In Table 6, it is observed that 2 workers, representing $13 \%$ of the sample, perceive that there is a small decline regarding environmental pollution in the studied area of the aforementioned mining company; 4 workers, representing $27 \%$, perceive that there is a regular decline in environmental pollution; and 9 workers, representing $60 \%$, perceive that there is a high decline in environmental pollution.

At the same time, an arithmetic mean of 5.27 was determined, which indicates an important pollution decline in the study area. A standard deviation with a variance of 2.07 with respect to the arithmetic mean has also been determined, demonstrating that the data are consistent.

\section{CONCLUSIONS}

1. Based on the results obtained, it was concluded that the iron ore dust control system reduces pollution and occupational exposure to iron dust.

2. The abovementioned system was proven effective.

3. As a result of the implementation of the iron ore dust mitigation system at the crushing plant, personnel exposure levels to particulate matter were reduced. Respirable and inhalable dust particles concentration levels do not exceed the established permissible limits of $3 \mathrm{mg} / \mathrm{m}^{3}$ and 10 $\mathrm{mg} / \mathrm{m}^{3}$, respectively.

4. The reduction of pollutant concentration in the crushing plant facilities, from $500 \mu \mathrm{g} / \mathrm{m} 3$ to a value within the maximum permissible limits as per air quality standards, was verified.

5. It has been demonstrated that it is possible to reduce the number of occupational diseases in workers.

\section{ACKNOWLEDGMENT}

To the Universidad Nacional Mayor de San Marcos, in particular, to the Unidad de Posgrado of the Facultad de Ingenieria Geológica, Minera, Metalúrgica y Geográfica ${ }^{\mathrm{TN} 2}$ of the same university for providing opportunities for professional growth.

TN2: Graduate Studies Unit of the School of Geological, Mining, Metallurgical and Geographical Engineering.

To our advisors, Oscar Tinoco Gomez and Vidal Aramburú Rojas, for their support and advice during the development of this research.

\section{REFERENCES}

[1] Aguilar, L. (2006). Contaminación ambiental. Retrieved from http://contaminacion-ambiente. blogspot.com.

[2] Anguita, F. \& Moreno, F. (1993). Procesos geológicos externos y geología ambiental. Madrid, Spain: Rueda.

[3] Bartual, J. \& Berenguer, M. J. (2001). NTP 607: Guías de calidad de aire interior: contaminantes químicos. Madrid, Spain: Instituto Nacional de Seguridad e Higiene en el Trabajo / Ministerio de Trabajo y Asuntos Sociales. Retrieved from https:// www.insst.es/documents/94886/326775/ 
ntp_607.pdf/0c6960b6-b461-4d21-9757e4ea03004327.

[4] Campos, I. (2000). Saneamiento ambiental. San José, Costa Rica: Editorial Universidad Estatal a Distancia.

[5] Castillo, H. (2008). Influencia antropogénica minera sobre la especie endémica Batrachophrynus macrostomus Peters, 1873 en peligro de extinción del lago Chinchaycocha (Pasco, Junín), 2007. (Doctoral thesis). Universidad Nacional de Trujillo, Peru. Retrieved from http://dspace.unitru.edu.pe/ bitstream/handle/UNITRU/5903/Tesis $\% 20$ Doctorado\%20-\%20HItlser\%20Castillo\%20 Paredes. . pdf? sequence=1\&isAllowed $=y$.

[6] García, A. \& Fernández, R. (December 6, 2008). Decreto Supremon. ${ }^{\circ} 007-2008-M I N A M$. Aprueban Reglamento de Organización y Funciones del Ministerio del Ambiente. Retrieved from http://www.minam.gob.pe/ wp-content/uploads/2013/09/ds_007-2008minam.pdf.

[7] Glave, M. \& Kuramoto, J. (2002). Minería, minerales y desarrollo sustentable en Perú. En Equipo MMSD América del Sur, Minería, minerales y desarrollo sustentable en América del Sur (pp. 529-591). London, United Kingdom: Centro de Investigación y Planificación del Medio Ambiente / Centro Internacional de Investigación para el Desarrollo / Iniciativa de Investigación sobre Políticas Mineras.

[8] Herrera, P. \& Millones, O. (2011). ¿Cuál es el costo de la contaminación ambiental minera sobre los recursos hídricos en el Perú? Informe final. Lima, Peru: Consorcio de Investigación Económica y Social / Departamento de Economía de la Pontificia Universidad Católica del Perú. Retrieved from http://www2.congreso. gob.pe/sicr/cendocbib/con3_uibd.nsf/FF 88A00731054C670525797A006117B0/\$FILE/ Informe_Final_Costo_Economico_de_la_ contaminacion_en_los_recursos_Hidrico.pdf.

[9] Hokama, D. (July 16, 1996). Resolución Ministerial n. ${ }^{\circ}$ 315-96 EM/VMM. Niveles máximos permisibles de elementos $y$ compuestos presentes en emisiones gaseosas provenientes de las unidades minero-metalúrgicas. Retrieved from http:// intranet2.minem.gob.pe/web/archivos/dgaam/ publicaciones/compendio99/rm315-96.pdf.

[10] Humala, O. \& Villena, P. (August 20, 2011). Reglamento de la leyn. ${ }^{\circ} 29783$, Ley de Seguridad y Salud en el Trabajo. Decreto Supremo n. ${ }^{\circ} 005-$
2012-TR. Retrieved from https://www.minagri. gob.pe/portal/download/pdf/seguridad-y-salud/ reglamento-ley29783.pdf.

[11] Humala, O. \& Villena, J. (April 25, 2012). Decreto Supremo $n .^{\circ}$ 005-2012-TR. Reglamento de la Ley $n .^{\circ} 29783$, Ley de Seguridad y Salud en el Trabajo. Retrieved from https://busquedas. elperuano.pe/normaslegales/reglamento-dela-ley-n-29783-ley-de-seguridad-y-salud-en-edecreto-supremo-n-005-2012-tr-781249-1/.

[12] Martínez, C., Quero, A., Isidro, J. \& Rego, G. (2001). Enfermedades pulmonares profesionales por inhalación de polvos inorgánicos. Jano, 61(1397), 34-39.

[13] Medina, F. (2008). Inventario de pasivos ambientales mineros. Lima, Peru: Proyecto de Reforma del Sector de Recursos Mineros del Perú.

[14] Ministerio de Energía y Minas (December 10, 1993). Reglamento sobre protección del medio ambiente. Decreto Supremo n. ${ }^{\circ}$ 059-93-EM. Retrieved from http:// www.minem.gob.pe/_legislacionM. php?idSector=4\&idLegislacion=5272.

[15] Moraga, P., Rivera, L. \& Soto, M. (2013). Evaluar y proponer la disminución de material particulado en la planta de chancado secundario-terciario en división El Teniente de Codelco Chile. (Degree thesis). Universidad Academia de Humanismo Cristiano, Chile. Retrieved from http://bibliotecadigital. academia.cl/handle/123456789/1236.

[16] Organismo de Evaluación y Fiscalización Ambiental (2014). Cartilla del abc de la fiscalización ambiental. Lima, Peru: Organismo de Evaluación y Fiscalización Ambiental. Retrieved from https://centroderecursos. cultura.pe/sites/default/files/rb/pdf/ Brochure $\% 20 \mathrm{EI} \% 20 \mathrm{ABC} \% 20 \mathrm{de} \% 20 \mathrm{la} \% 20$ fiscalizacion\%20ambiental.pdf.

[17] Vega, D. (2007). El origen de polvos y su mitigación en la minería. Trabajo de investigación. Monografias.com. Retrieved from https://www.monografias.com/trabajos53/ contaminacion-polvos-mineria/contaminacionpolvos-mineria.shtml.

[18] Warner, P. (1981). Análisis de los contaminantes del aire. Madrid, Spain: Paraninfo. 